\title{
Characteristics of rhegmatogenous retinal detachment in pseudophakic and phakic eyes
}

${ }^{1}$ Department of

Ophthalmology, St Thomas' Hospital, London, UK

${ }^{2}$ Department of Ophthalmology, King's College London, St Thomas' Hospital Campus, London, UK

${ }^{3}$ School of Computing, University of East London, London, UK

Correspondence: TH Williamson, Department of Ophthalmology, St Thomas Hospital, Lambeth Palace Road, Westminster Bridge Road, London SE1 7EH, UK Tel: +44 (0)207 188 4320; Fax: +44 (0)207 9228157. E-mail: Tom@

retinasurgery.co.uk

Received: 7 September 2011

Accepted in revised form: 23 April 2012

Published online: 8 June 2012

\begin{abstract}
Aims To investigate whether pseudophakic and phakic rhegmatogenous retinal detachment (RRD) patterns differ. Methods Retrospective review of electronic database of patients, aged 50 years or over, presenting to our vitreoretinal service. Data included baseline characteristics, digital drawings, and outcomes. Retinal drawings were analysed in a masked fashion for site, size, and number of retinal breaks. Comparisons were made between the following groups and subgroups: pseudophakic eyes, phakic eyes, phakic eyes with cataract, and phakic eyes without cataract.

Results Of 500 eyes included, 146 were pseudophakic; 177 of the phakic eyes had cataract. The following were significant by univariate analysis: pseudophakic patients were older than phakic patients in general, but the same age as patients with cataract; in the pseudophakic group, there were lower proportions of females and of patients presenting with vitreous haemorrhage or with large or superotemporal breaks; higher proportions of pseudophakic eyes had small breaks and inferonasal breaks. Some differences remained significant when comparing pseudophakia eyes with cataract. Multivariate analysis comparing pseudophakia with phakia confirmed a lower chance in pseudophakia of large breaks, vitreous haemorrhage and superotemporal breaks, but higher chance of detached inferior breaks. Some variables were age dependent. Conclusion Differences were found between pseudophakic and phakic RRD patterns. These suggest special pathogenetic mechanisms in pseudophakic retinal detachment, which could help explain increased incidences of RRD after cataract surgery.
\end{abstract}

OAR Mahroo ${ }^{1,2}$, R Dybowski $^{3}$, R Wong $^{1}$ and TH Williamson'
Eye (2012) 26, 1114-1121; doi:10.1038/eye.2012.112; published online 8 June 2012

Keywords: rhegmatogenous retinal detachment; retinal break; cataract; pseudophakia; phacoemulsification

\section{Introduction}

Cataract surgery has evolved rapidly in the last 40 years, progressing from intracapsular surgery and postoperative aphakia to extracapsular surgery with implantation of anterior chamber intraocular lenses, to phacoemulsification with continuous curvilinear capsulorhexis and insertion of posterior chamber lenses. This has led to a reduction in complications and improved postoperative outcome. The relationship between cataract surgery and rhegmatogenous retinal detachment (RRD) has been studied using data from Medicare and other insurance information, ${ }^{1-3}$ Scandinavian public health data, ${ }^{4,5}$ and large population-based studies such as The Rochester Study. ${ }^{6,7}$ These studies suggest an increased risk of retinal detachment associated with cataract operations despite progress in surgical technique. The 4-year incidence of retinal detachment after all cataract extractions has been reported as $1.17 \%$, with higher rates in cases of vitreous loss $(4.9 \%)$ and a lower rate with the use of phacoemulsification $(0.4 \%){ }^{8}$ This is higher than would be expected in the normal population: studies have reported an annual incidence of retinal detachment of between 6.3 and 17.9 per $100000 .^{9}$

Epidemiological data, however, do not discriminate between an association of cataract with retinal detachment or cataract surgery with retinal detachment. It is possible that the presence of a cataract itself is a marker for 
higher risk of retinal detachment. It has been shown, for example, that patients with a retinal detachment in a pseudophakic eye have a significant risk of an RRD in the other eye even if that eye does not undergo cataract surgery. ${ }^{10}$

A potential strategy for exploring the link between retinal detachment and cataract surgery is to examine the clinical pattern of retinal detachment, for example, position, size, and type of retinal breaks, and to see if this differs between pseudophakic and phakic eyes. If differences in pattern are observed, this may suggest a difference in the pathogenetic mechanism. Previous studies have been performed in this regard in the 1960s and 1970s when intracapsular cataract surgery and aphakia were common. ${ }^{11-15}$ However, to our knowledge, there are no studies in the English language literature on the pattern of retinal detachment in the era of phacoemulsification, an operation regarded as less traumatic to the internal structures of the eye than both intracapsular and extracapsular surgery, and leading to pseudophakia, usually with an intact capsular bag, rather than aphakia.

\section{Materials and methods}

Data from all patients attending a single author's (THW) vitreoretinal surgery service were prospectively entered into an electronic patient record (Microsoft Access, available with the book Vitreoretinal Surgery $\left.{ }^{16}\right)$. The electronic patient record requires recording of retinal detachment characteristics and digital drawings of the retinal detachment. The duration of symptoms, type of breaks, number of breaks, presence of proliferative vitreoretinopathy (PVR) according to the revised Retinal Society classification, ${ }^{17}$ extent of retinal detachment, foveal status, presence of vitreous haemorrhage, and visual acuity (determined using Snellen charts, but converted to $\log \mathrm{MAR}$ ) were recorded. In addition, preoperative data on the status of the lens (including presence of visually significant cataract or pseudophakia) and date of prior cataract surgery were recorded. Ethical approval for the database study was obtained from the local research ethics committee (Guys and St Thomas' Hospitals Trust).

For patients who developed retinal detachment in both eyes, only the first eye was included in the present study. Patients under 50 years of age, or with aphakia, anterior chamber lens implant, giant retinal tear, retinal dialysis, macular hole related RRD, retinoschisis-related RRD, and dislocated lens nucleus during cataract surgery were excluded.

Retinal drawings were reviewed by an observer (THW) who was masked to the clinical data of the patient. Drawings were divided into four quadrants centred at the fovea: superotemporal, superonasal, inferotemporal, and inferonasal. The observer recorded whether the eye had any breaks (true or false) in any of the four quadrants. This method was used to avoid any conflict of dependant variables that would arise by counting the numbers of breaks in each quadrant and using these as independent variables, a procedure that has been used in prior studies, ${ }^{18,19}$ resulting in erroneous statistical analysis, as multiple breaks in the same eye are not independent variables. The presence of inferior breaks was recorded, specifically noting whether breaks were present between 5 and 7 o'clock (true or false), or at 4-5 or 7-8 o' clock (true or false), and whether these were in flat or detached retina. Such breaks can present particular challenges in surgical management, compared with breaks elsewhere, hence this subdivision was not applied to superior, nasal, or temporal breaks. Eyes were regarded as having an anterior break if a break was identified anterior to a line halfway between the equator and the ora serrata. Eyes had a posterior break if a break was seen posterior to a line of similar distance posteriorly from the equator as the ora serrata was anterior to the equator.

In addition, breaks were regarded as small, medium, and large according to their longest meridian and related to the optic disc diameter. A small break was defined as $<0.5$ disc diameter; medium break size was 0.5-2 disc diameters; and large $>2$ disc diameters. Eyes were recorded (true or false) as having small, medium, or large breaks. Thus, a single eye could have small, medium, and large breaks if all sizes were present.

Data were analysed using statistical software for univariate analysis (Analyse-it, Cambridge, UK) and multivariate logistic regression analysis (using the $\mathrm{R}$ statistical language). Pair-wise comparisons for each variable were made between pseudophakic eyes ('pseudophakia') and phakic eyes ('phakia'), between pseudophakic eyes and phakic eyes with cataract ('phakic cataract'), and between phakic eyes without cataract ('phakic non-cataract') and phakic eyes with cataract ('phakic cataract'). Means were compared using $t$-test and proportions using Fisher's exact test.

\section{Results}

A total of 535 eyes from 535 patients were included in the study. Thirty-five drawings were missing or inadequate, so these eyes were excluded from analysis. Of the 500 patients, 354 were phakic, of whom 177 had visually significant cataract, and 146 were pseudophakic. The duration since cataract surgery was known for 130 of the 146 pseudophakic eyes, and ranged from 3 days to 20 years (median 1.94 years)-see Figure 1. There was a history of YAG capsulotomy in $14.0 \%$ of the 


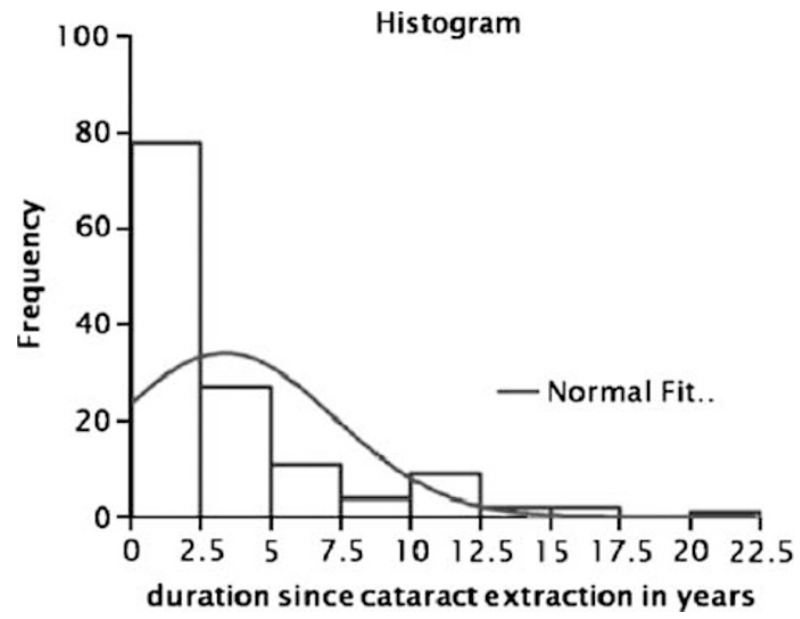

Figure 1 Histogram of the duration between cataract surgery and operation for retinal detachment in years for the 130 pseudophakic eyes for which this was known.

pseudophakic eyes and a history of cataract surgery complicated by posterior capsular tear in $10.5 \%$.

However, these numbers may represent under-estimates as the complete records of previous ocular history were not always available, particularly as referrals were primarily from other hospitals to the tertiary vitreoretinal service.

The following data were for the group as a whole: median duration of vision loss was 8 days; median follow-up was 10 months; the right eye was involved in $53 \%$; the fovea was off at presentation in 58\%; the mean number of breaks was 2.5; multiple breaks (more than one break) were present in 55\%; and no hole was found in $1 \%$. The rate of PVR at presentation of grades B or C was $14 \%$. Surgical success rates were similar between the groups with primary success rate of $85 \%$ and secondary success rate of $96 \%$.

\section{Univariate analysis}

Table 1 shows the results of univariate comparisons between the different groups. The following differences were significant: pseudophakic patients were older than phakic patients in general, but the same age as phakic patients with cataract; in the pseudophakic group, there was a lower proportion of females, a lower proportion presenting with vitreous haemorrhage, and a lower proportion with large breaks or superotemporal breaks; however, a higher proportion of pseudophakic eyes had small breaks and inferonasal breaks compared with the other groups. See Figure 2 (upper panel) for comparison of proportions with large or small breaks and superotemporal or inferonasal breaks in pseudophakia and phakia. Several of these differences were seen also when comparing pseudophakic eyes
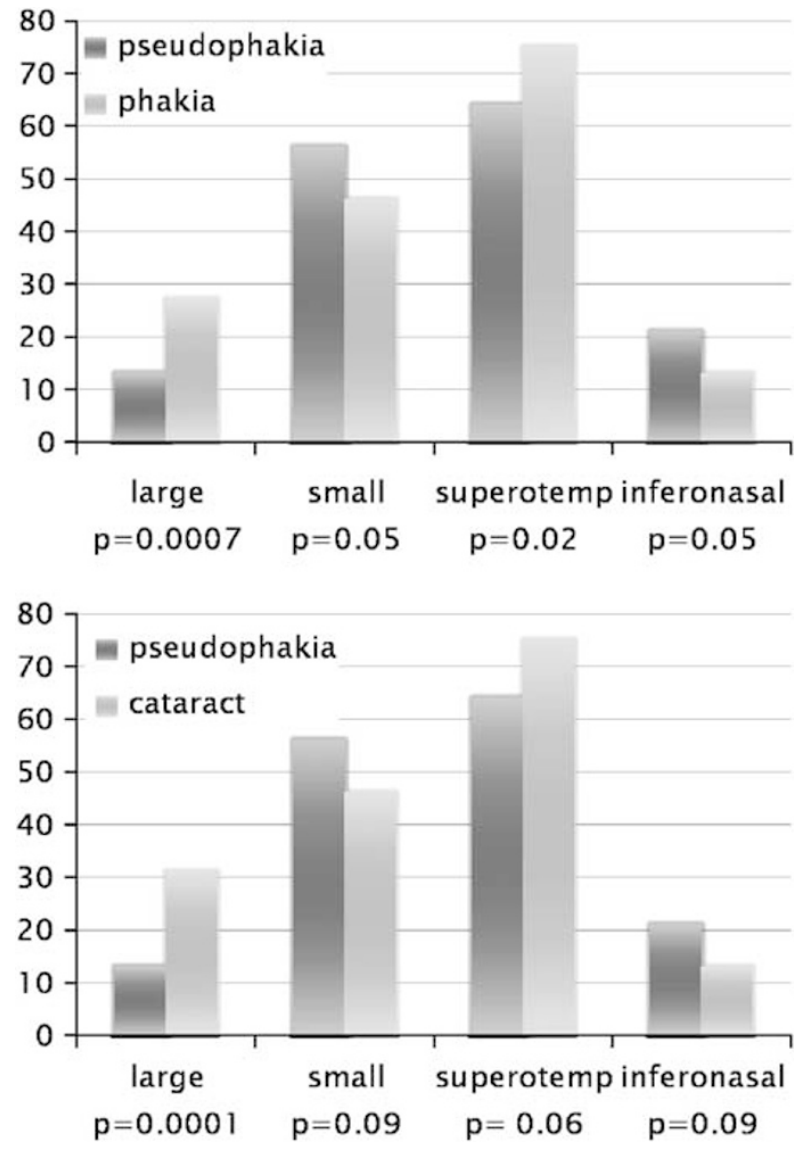

Figure 2 Comparison of proportions of patients (\%) with large, small, superotemporal, or inferonasal breaks in pseudophakic $v s$ phakic eyes (upper panel) and in pseudophakic vs phakic eyes with cataract (lower panel). $P$-values found by univariate analysis.

specifically with phakic eyes with cataract (Table 1), but statistical significance was not reached in all cases. This may be expected as numbers are smaller for this subgroup comparison, although a trend is still discernible.

The lower panel of Figure 2 compares the same variables as in the upper panel, but between the pseudophakic and phakic cataract groups. Here, the same trend is observed: pseudophakic eyes have a lower proportion with large or superotemporal breaks and higher proportions with small or inferonasal breaks, but the numbers fail to reach statistical significance for three out of the four comparisons.

Most variables were similar in phakic cataract and phakic non-cataract groups, except for the following where statistically significant differences were observed (see Table 1): phakic non-cataract patients were younger (as mentioned earlier) and had better presenting and final acuity; a lower proportion presented with the fovea detached or with PVR; and a higher proportion 
Table 1 Features of retinal detachments in pseudophakic and phakic eyes

\begin{tabular}{|c|c|c|c|c|c|c|c|}
\hline Variable & $\begin{array}{c}\text { Pseudophakic } \\
\text { eyes } \\
(\mathrm{n}=146)\end{array}$ & $\begin{array}{c}\text { All phakic } \\
\text { eyes } \\
(\mathrm{n}=354)\end{array}$ & $\begin{array}{l}\text { Phakic } \\
\text { eyes with } \\
\text { cataract } \\
(\mathrm{n}=177)\end{array}$ & $\begin{array}{l}\text { Phakic eyes } \\
\text { without } \\
\text { cataract } \\
(\mathrm{n}=177)\end{array}$ & $\begin{array}{c}\text { P-value } \\
\text { (pseudophakic } \\
\text { vs phakic) }\end{array}$ & $\begin{array}{c}\text { P-value } \\
\text { (pseudophakic } \\
\text { vs phakic } \\
\text { cataract) }\end{array}$ & $\begin{array}{c}\text { P-value } \\
\text { (phakic cataract } \\
\text { vs phakic without } \\
\text { cataract) }\end{array}$ \\
\hline \multicolumn{8}{|l|}{ Baseline characteristics } \\
\hline Age in years (mean $\pm \mathrm{sD})$ & $68.9 \pm 9.9$ & $64.2 \pm 9.2$ & $68.3 \pm 9.9$ & $60.2 \pm 6.3$ & $<0.0001^{*}$ & 0.570 & $<0.0001^{*}$ \\
\hline Sex ( $\%$ female) & 31.5 & 41.5 & 42.9 & 40.1 & $0.045^{*}$ & $0.046^{*}$ & 0.666 \\
\hline \multicolumn{8}{|l|}{ Presenting features } \\
\hline $\begin{array}{l}\text { Presenting } \log M A R \text { acuity } \\
(\text { mean } \pm \mathrm{SD})\end{array}$ & $0.96 \pm 0.74$ & $0.90 \pm 0.77$ & $1.11 \pm 0.75$ & $0.68 \pm 0.74$ & 0.404 & 0.073 & $<0.0001^{*}$ \\
\hline $\begin{array}{l}\text { Duration of visual loss in days } \\
\text { (mean } \pm \mathrm{sD})\end{array}$ & $\begin{array}{l}15.5 \pm 23.6 \\
(n=116)\end{array}$ & $\begin{array}{l}31.4 \pm 94.5 \\
(n=276)\end{array}$ & $\begin{array}{c}34.2 \pm 80.8 \\
(n=140)\end{array}$ & $\begin{array}{l}28.5 \pm 107 \\
(n=136)\end{array}$ & 0.074 & $0.017^{*}$ & 0.619 \\
\hline $\begin{array}{l}\text { Vitreous haemorrhage at } \\
\text { presentation? }(\%)\end{array}$ & 6.8 & 17.2 & 16.4 & 18.1 & $0.002^{*}$ & $0.013^{*}$ & 0.779 \\
\hline Presence of PVR (\%) & 14.4 & 13.6 & 19.8 & 7.3 & 0.909 & 0.260 & $0.001^{*}$ \\
\hline Number of breaks (mean \pm SD) & $2.5 \pm 2.3$ & $2.7 \pm 2.5$ & $2.7 \pm 2.6$ & $2.6 \pm 2.3$ & 0.638 & 0.607 & 0.812 \\
\hline Fovea off? (\%) & 63.7 & 55.4 & 67.2 & 56.5 & 0.105 & 0.583 & $<0.0001^{*}$ \\
\hline \multicolumn{8}{|l|}{ Presence of different sized breaks } \\
\hline Small breaks present? (\%) & 56.2 & 46.0 & 46.3 & 45.8 & $0.050^{*}$ & 0.099 & 1.00 \\
\hline Medium breaks present? (\%) & 60.3 & 59.0 & 52.0 & 66.1 & 0.879 & 0.167 & $0.009^{*}$ \\
\hline Large breaks present? (\%) & 13.0 & 27.1 & 31.6 & 22.6 & $0.0007^{*}$ & $0.0001^{*}$ & 0.073 \\
\hline \multicolumn{8}{|l|}{ Break position by quadrant } \\
\hline Superotemporal break? (\%) & 64.4 & 74.9 & 74.6 & 75.1 & $0.025^{*}$ & 0.062 & 1.00 \\
\hline Inferotemporal break? (\%) & 26.7 & 31.9 & 32.8 & 31.1 & 0.296 & 0.289 & 0.820 \\
\hline Superonasal break? (\%) & 38.4 & 38.4 & 40.1 & 36.7 & 1.00 & 0.836 & 0.585 \\
\hline Inferonasal break? (\%) & 21.2 & 13.6 & 13.6 & 13.6 & $0.049^{*}$ & 0.094 & 1.00 \\
\hline \multicolumn{8}{|l|}{ Anterior or posterior break position } \\
\hline Anterior break? (\%) & 28.1 & 21.2 & 19.2 & 23.2 & 0.125 & 0.081 & 0.435 \\
\hline Posterior break? (\%) & 13.0 & 16.7 & 16.9 & 16.4 & 0.376 & 0.410 & 1.00 \\
\hline \multicolumn{8}{|l|}{ Position of inferior flat breaks } \\
\hline Flat break $4-5$ or $7-8$ o'clock? (\%) & 1.4 & 4.8 & 3.4 & 6.2 & 0.101 & 0.427 & 0.320 \\
\hline Flat break 5-7 o'clock? (\%) & 8.2 & 8.5 & 8.5 & 8.5 & 1.00 & 1.00 & 1.00 \\
\hline \multicolumn{8}{|l|}{ Position of inferior breaks involved in $R D$} \\
\hline Break in RD $4-5$ or $7-8$ o' clock? (\%) & 9.6 & 8.5 & 7.9 & 9.0 & 0.807 & 0.735 & 0.849 \\
\hline Break in RD 5-7 o'clock? (\%) & 13.0 & 7.3 & 7.9 & 6.8 & 0.071 & 0.186 & 0.839 \\
\hline \multicolumn{8}{|l|}{ Outcome measures } \\
\hline Years of follow-up (mean \pm SD) & $0.72 \pm 1.10$ & $0.92 \pm 1.09$ & $0.98 \pm 1.19$ & $0.85 \pm 0.98$ & 0.071 & $0.045^{*}$ & 0.267 \\
\hline $\begin{array}{l}\log M A R \text { acuity at last } \\
\text { follow-up (mean } \pm \text { sD) }\end{array}$ & $0.46 \pm 0.56$ & $0.44 \pm 0.52$ & $0.50 \pm 0.55$ & $0.39 \pm 0.48$ & 0.822 & 0.487 & $0.046^{*}$ \\
\hline Any RD at final follow-up? (\%) & 4.8 & 4.0 & 5.1 & 2.8 & 0.833 & 1.00 & 0.414 \\
\hline Oil in at final follow-up? $(\%)$ & 9.6 & 5.4 & 7.3 & 3.4 & 0.132 & 0.599 & 0.156 \\
\hline Phthisis at final follow-up? (\%) & 1.4 & 0.0 & 0.0 & 0.0 & 0.170 & 0.407 & 1.00 \\
\hline
\end{tabular}

Asterisk denotes significance at the $5 \%$ level. Total numbers of eyes given in the top row apply to all rows except for 'duration of visual loss' where numbers are smaller (displayed in each cell) due to missing data for some eyes.

had medium-sized breaks compared with the phakic cataract group.

\section{Multivariate analysis}

Multivariate analysis confirmed a lower chance of large breaks in pseudophakia compared with phakia.
Vitreous haemorrhage and superotemporal breaks were less common in pseudophakia. Detached inferior breaks between 5 and 7 o'clock hours were more common in pseudophakia. Pseudophakic patients were more likely to have RRD involving four quadrants. See Table 2 for odds ratios (ORs). After multivariate analysis, differences for small breaks were not significant. 
Table 2 Multivariate analysis comparing pseudophakia with phakia

Odds ratio

(95\% confidence interval)

Large breaks

$0.43(0.25,0.73)$

Small breaks

Not significant

Vitreous haemorrhage

Superotemporal breaks

Inferior break 5-7 o'clock in RRD

Four quadrant RRD

Abbreviation: RRD, rhegmatogenous retinal detachment.


Figure 3 Odds ratio for the presence of inferonasal break (left panel) or foveal detachment (right panel) in pseudophakia compared with phakia, as a function of age (in years), found by multivariate analysis. Dotted lines plot $95 \%$ confidence intervals.

Mulitivariate comparison between pseudophakic eyes and phakic eyes with cataract confirmed that there was a higher chance of large breaks in the phakic cataract group (OR 2.36, 95\% confidence interval (CI): 1.51, 3.69).

Multivariate comparison between the two phakic groups (phakic cataract and phakic non-cataract) showed that, if cataract were present, there was a higher chance of fovea off (OR 2.60, 95\% CI: 1.69, 4.00) and a lower chance of medium breaks (OR $0.59,95 \%$ CI: $0.39,0.90$ ) or retinal detachment in only 1,2, or 3 quadrants (OR 0.46 , 95\% CI: 0.26, 0.82). No other comparisons (including presence of large breaks) were significant by multivariate analysis between the two phakic groups.

Two variables were age dependent: the presence of inferonasal breaks and presence of foveal detachment at presentation. Figure 3 shows how the OR changes with age group (together with CIs): inferonasal breaks were significantly more likely in pseudophakia overall, but the OR increased with age; foveal detachment was also more likely in pseudophakia, but here the OR declined with age. None of the other variables showed such age dependence.

\section{Discussion}

This study aimed to explore differences in retinal detachment patterns in pseudophakia compared with phakia. Several differences were statistically significant, and a similar trend was discernible even when comparing pseudophakic patients specifically with phakic patients with cataract, but statistical significance was not achieved as frequently. Some of the differences were also observed by multivariate analysis.

A higher proportion of males was found in all groups, agreeing with epidemiological studies that have shown a greater incidence of retinal detachment in males. ${ }^{20}$ The male predilection for RRD was more pronounced in pseudophakic RRD despite the higher number of cataract operations performed on female patients in the general population and most studies showing more presentations of symptomatic posterior vitreous detachments in females. ${ }^{21-23}$ This difference between the sexes remains unexplained at present.

Age can affect the pattern of retinal detachment, ${ }^{24}$ so steps were taken to control this. Patients under 50 years of age were excluded so that the pseudophakic and phakic groups would be comparable, and indeed the pseudophakic and phakic cataract groups had the same mean age. Also, multivariate analysis was used to control for age dependence: the ORs for only two variables were found to show age dependence (Figure 3).

Patients who were pseudophakic were less likely to have vitreous haemorrhage as a presenting feature, and this may be related to the smaller number of eyes with large breaks seen in this group. The most common site for breaks was superotemporal in all groups but this area was less often involved in pseudophakia than in phakia. Inferonasal breaks were more common in pseudophakia by univariate analysis, and there was a greater chance of detached breaks inferiorly between 5 and 7 o'clock in pseudophakia after multivariate analysis.

Many variables were similar between phakic patients with cataract and phakic patients without cataract, although a lower proportion of eyes with medium-sized breaks, a greater proportion with PVR and a greater proportion with detached foveas were observed in patients with cataract by univariate analysis. Multivariate analysis confirmed that the presence of cataract in a phakic eye gave a lower chance of mediumsized breaks and a higher chance of detached fovea. The biological basis for this is unclear. Phakic eyes with cataract were more likely to have large, and less likely to have medium, breaks than those without cataract, whereas the proportions of small breaks were similar in both groups (Table 1), and so one would postulate that eyes with larger breaks would be likely to have progressed to foveal detachment by presentation.

In our study, $\sim 10.5 \%$ of pseudophakic patients had undergone cataract extraction complicated by posterior capsular tear, and $14 \%$ had undergone a YAG capsulotomy. Such events could be important in the 
development of the pseudophakic RRD pattern that we observe. However, subgroup analysis of these data within our study would be unlikely to yield reliable conclusions given that the numbers are small and there was uncertainty over the actual proportions of eyes that had undergone YAG capsulotomy or complicated cataract extraction, because of lack of availability of full records of prior ocular history in many cases. By excluding patients with anterior chamber lens implants, aphakia, and dislocated nuclear lens fragments, we hoped to reduce the influence of severely complicated cataract operations on the results.

Koo et al ${ }^{25}$ compared retinal detachments in 206 phakic and 37 pseudophakic eyes. The male preponderance was similarly more marked in the pseudophakic group. High myopia and peripheral retinal degenerations were more common in the phakic group. The proportion with two or more breaks was greater in the phakic group. The proportion with detachment involving three or four quadrants, or involving the macula, was greater in the pseudophakic group, similar to the findings of our study. However, their study differed from ours as it included all age groups, and had a relatively small pseudophakic group (37 eyes), of which significant proportions had posterior capsular rupture $(40.5 \%)$ and no break found (27\%). The presence of high myopia was not recorded in our study, and may have relevance in affecting the pattern of detachment.

Interestingly, previous studies of aphakic retinal detachment show a pattern of difference between aphakic and phakic retinal detachment, which bears some similarities to the present study. Phillips ${ }^{18}$ in 1963 found that a greater proportion of aphakic retinal detachments had inferior breaks and more peripheral breaks compared with phakic eyes. Norton ${ }^{26}$ reported in 1964 that compared with phakic eyes, aphakic eyes were less likely to have superotemporal breaks, or to present with the macula attached or vitreous haemorrhage. Everett and Katzin ${ }^{27}$ in 1968 found that aphakic eyes had a lower percentage of females, a lower proportion with superotemporal breaks, and a higher proportion with inferonasal breaks compared with phakic eyes, and these findings were also seen in the large series of Ashrafzadeh et al. ${ }^{19}$ The latter study found that more aphakic eyes had oral and fewer had equatorial breaks than the phakic group. Aphakic eyes were in addition more likely to have total retinal and macular detachment, and were slightly less likely to present with vitreous haemorrhage, although the difference here was not statistically significant.

The older studies used a system of counting the number of breaks in each quadrant, providing a total number of breaks in each quadrant over a cohort.
Applying statistical analysis using this methodology is more complicated because breaks from the same eye are not independent variables. The use of this method may explain the increased percentages described in these studies compared with the present study in which the eye was only classified as having breaks present or absent in each quadrant or area of the retina, regardless of the number.

Yoshida et $a l^{28}$ compared retinal detachment characteristics in aphakic and pseudophakic eyes (following intracapsular or extracapsular extraction) in 1992. They found that the aphakic group tended to have smaller and more peripheral breaks, leading the authors to speculate that pseudophakic retinal detachments may be more similar to those seen in phakic eyes than aphakic eyes. However, they found no significant difference in the quadrantal location of retinal breaks between the groups.

Previous population-based studies suggest a reduction in the incidence of pseudophakic retinal detachment with phacoemulsification compared with intracapsular cataract extraction. ${ }^{8}$ Our study has shown a pattern of pseudophakic RRD, after phacoemulsification, which has some striking similarities to the aphakic retinal detachments seen in the 1960s and 70s. It is possible that there is a disproportionate effect of some patients undergoing YAG capsulotomy or complicated cataract extraction on the position of retinal breaks. However, it is quite plausible that the physical effect of replacing a large cataractous lens with a small plastic intraocular lens causes changes in vitreous anatomy that influences the type and position of retinal break. It has been postulated that cataract surgery is more likely to induce an anomalous posterior vitreous detachment (relevant studies reviewed by Coppe and Lapucci ${ }^{29}$ ), thus conferring an increased risk of RRD. We suggest that this may also account for the differences in pattern of RRD shown in our study. The greater proportions of inferonasal breaks seen in pseudophakic eyes in our study, or aphakic eyes in previous studies, is interesting. Cataract surgery incisions are usually superior or temporal, but whether or how this might contribute to vitreous dynamics and patterns of retinal break is unknown.

Some potential limitations of the present study deserve mention. Although all retinal drawings were analysed consistently by a single author, the drawings themselves were made by different surgeons, who may have estimated break size differently. Visualisation of breaks may also differ among patient groups: the presence of an intraocular lens may make breaks appear smaller or affect their perceived position; breaks may also be more difficult to detect in the pseudophakic eye because of obscuration by the lens edge or by capsular opacification. 
However, the presence of cataract, or vitreous haemorrhage (which was more common in phakic eyes), may have a similar effect. The presence or absence of lattice degeneration, which could have a bearing on the pattern of retinal break or detachment, was also not recorded.

A further limitation concerns division of the phakic group into those with or without visually significant cataract. Given that a spectrum of lens opacity exists, such distinction may be somewhat subjective and arbitrary. The study has partly assumed that the only difference between the phakic cataract group and the pseudophakic group is the event of the cataract operation. It is quite possible that there are other differences between these eyes that explain why some had undergone cataract extraction and others had not, and it may be that these underlying differences could explain the different RRD patterns.

In summary, patients with pseudophakic retinal detachment appear to have a pattern of retinal detachment which differs from that seen in phakic patients and also from that seen in phakic patients with cataract. This suggests that the event of phacoemulsification cataract surgery itself may contribute to a distinct pathogenetic mechanism for retinal detachment in these patients. Although patterns are broadly similar between the groups, the presence of statistically significant differences and of similar trends observed in both comparisons (pseudophakia vs phakia and pseudophakia vs phakic cataract), together with the similarities to historical descriptions of aphakic retinal detachment after intracapsular cataract surgery, suggest that cataract surgery itself could be a determining factor.

\section{Summary}

\section{What was known before}

- The incidence of retinal detachment is higher in eyes that have undergone cataract surgery. Older studies of aphakic retinal detachment showed differences in the patterns of detachments between aphakic and phakic eyes.

\section{What this study adds}

- The patterns of retinal detachment differ between pseudophakic and phakic eyes (and also between pseudophakic and phakic eyes with cataract).

- The patterns of detachment in pseudophakic eyes bear some similarity to patterns seen previously in aphakic eyes.

- The results are consistent with the notion that the event of cataract surgery itself, rather than simply the presence of cataract, increases the risk of retinal detachment and affects the pattern of breaks.

\section{Conflict of interest}

The authors declare no conflict of interest.

\section{Acknowledgements}

Financial support was received from the charity Eyehope (UK registered charity 1119866). The funding organisation had no role in the design or conduct of this research.

\section{References}

1 Javitt JC, Vitale S, Canner JK, Krakauer H, McBean AM, Sommer A. National outcomes of cataract extraction. I. Retinal detachment after inpatient surgery. Ophthalmology 1991; 98: 895-902.

2 Javitt JC, Tielsch JM, Canner JK, Kolb MM, Sommer A, Steinberg EP. National outcomes of cataract extraction. Increased risk of retinal complications associated with Nd:YAG laser capsulotomy. The Cataract Patient Outcomes Research Team. Ophthalmology 1992; 99: 1487-1497.

3 Sheu SJ, Ger LP, Ho WL. Late increased risk of retinal detachment after cataract extraction. Am J Ophthalmol 149(1): 113-119.

4 Boberg-Ans G, Villumsen J, Henning V. Retinal detachment after phacoemulsification cataract extraction. J Cataract Refract Surg 2003; 29(7): 1333-1338.

5 Boberg-Ans G, Henning V, Villumsen J, la Cour M. Longterm incidence of rhegmatogenous retinal detachment and survival in a defined population undergoing standardized phacoemulsification surgery. Acta Ophthalmol Scand 2006; 84: 613-618.

6 Erie JC, Raecker ME, Baratz KH, Schleck CD, Robertson DM. Risk of retinal detachment after cataract extraction, 1980-2004: a population-based study. Trans Am Ophthalmol Soc 2006; 104: 167-175.

7 Lois N, Wong D. Pseudophakic retinal detachment. Surv Ophthalmol 2003; 48(5): 467-487.

8 Bradford JD, Wilkinson CP, Fransen SR. Pseudophakic retinal detachments. The relationships between retinal tears and the time following cataract surgery at which they occur. Retina 1989; 9(3): 181-186.

9 Mitry D, Charteris DG, Fleck BW, Campbell H, Singh J. The epidemiology of rhegmatogenous retinal detachment: geographical variation and clinical associations. $\mathrm{Br} \mathrm{J}$ Ophthalmol 2010; 94: 678-684.

10 Sharma MC, Chan P, Kim RU, Benson WE. Rhegmatogenous retinal detachment in the fellow phakic eyes of patients with pseudophakic rhegmatogenous retinal detachment. Retina 2003; 23: 37-40.

11 Menezo JL, Frances J, Reynolds RS. Number and shape of tears in aphakic retinal detachment: its relationship with different surgical techniques of cataract extraction. Mod Probl Ophthalmol 1977; 18: 457-463.

12 Snyder WB, Bernstein I, Fuller D, Hutton WL, Vaiser A. Retinal detachment and pseudophakia. Ophthalmology 1979; 86(2): 229-241.

13 Ramos M, Kruger EF, Lashkari K. Biostatistical analysis of pseudophakic and aphakic retinal detachments. Semin Ophthalmol 2002; 17(3-4): 206-213. 
14 McDonnell PJ, Patel A, Green WR. Comparison of intracapsular and extracapsular cataract surgery. Histopathologic study of eyes obtained postmortem. Ophthalmology 1985; 92(9): 1208-1225.

15 Tuft SJ, Minassian D, Sullivan P. Risk factors for retinal detachment after cataract surgery: a case-control study. Ophthalmology 2006; 113(4): 650-656.

16 Williamson TH. Vitreoretinal Surgery. Springer: Berlin, 2008.

17 Machemer R, Aaberg TM, Freeman HM, Irvine AR, Lean JS, Michels RM. An updated classification of retinal detachment with proliferative vitreoretinopathy. Am J Ophthalmol 1991; 112: 159-165.

18 Phillips CI. Distribution of breaks in aphakic and 'Senile' eyes with retinal detachments. Br J Ophthalmol 1963; 47: 744-752.

19 Ashrafzadeh MT, Schepens CL, ElzeneinyII, Moura R, Morse P, Kraushar MF. Aphakic and phakic retinal detachment. I. Preoperative findings. Arch Ophthalmol 1973; 89(6): 476-483.

20 Mitry D, Charteris D, Yorston D, Siddiqui MA, Campbell H, Murphy AL et al. The clinical epidemiology and socioeconomic associations of primary retinal detachment in Scotland: a two-year prospective population based study. Invest Ophthalmol Vis Sci 2011; 52: 2551-2555.

21 Tanner V, Harle D, Tan J, Foote B, Williamson TH, Chignell $\mathrm{AH}$. Acute posterior vitreous detachment: the predictive value of vitreous pigment and symptomatology. $\mathrm{Br} \mathrm{J}$ Ophthalmol 2000; 84: 1264-1268.

22 Chuo JY, Lee TY, Hollands H, Morris AH, Reyes RC, Rossiter JD et al. Risk factors for posterior vitreous detachment: a case-control study. Am J Ophthalmol 2006; 142(6): 931-937.

23 Richardson PS, Benson MT, Kirkby GR. The posterior vitreous detachment clinic: do new retinal breaks develop in the six weeks following an isolated symptomatic posterior vitreous detachment? Eye 1999; 13(Pt 2): 237-240.

24 Wilkinson CP. Retinal detachments in the elderly. Retina 1995; 15: 220-223.

25 Koo YM, Lee MS, Yoon IH. Comparsion of clinical findings between phakic and pseudophakic retinal detachment J Korean Ophthalmol Soc 1998; 39: 2995-3002.

26 Norton EWD. Retinal detachment in aphakia. Am J Ophthalmol 1964; 58: 111-124.

27 Everett WG, Katzin D. Meridional distribution of retinal breaks in aphakic retinal detachment. Am J Ophthalmol 1968; 66: 928-932.

28 Yoshida A, Ogasawara H, Jalkh AE, Sanders RJ, McMeel JW, Schepens CL. Retinal detachment after cataract surgery. Surgical results. Ophthalmology 1992; 99(3): 460-465.

29 Coppe AM, Lapucci G. Posterior vitreous detachment and retinal detachment following cataract extraction. Curr Opin Ophthalmol 2008; 19: 239-242. 